\title{
Autologous fat grafting: use of closed syringe microcannula system for enhanced autologous structural grafting
}

This article was published in the following Dove Press journal:

Clinical, Cosmetic and Investigational Dermatology

6 April 2013

Number of times this article has been viewed

\author{
Robert W Alexander \\ David B Harrell ${ }^{2}$ \\ 'Department of Surgery, School of \\ Medicine and Dentistry, University \\ of Washington, Seattle, WA, USA; \\ ${ }^{2}$ Harvest-Terumo Inc, Plymouth, MA, \\ USA
}

Objectives: Provide background for use of acquiring autologous adipose tissue as a tissue graft and source of adult progenitor cells for use in cosmetic plastic surgery. Discuss the background and mechanisms of action of closed syringe vacuum lipoaspiration, with emphasis on accessing adipose-derived mesenchymal/stromal cells and the stromal vascular fraction (SVF) for use in aesthetic, structural reconstruction and regenerative applications. Explain a proven protocol for acquiring high-quality autologous fat grafts (AFG) with use of disposable, microcannula systems.

Design: Explain the components and advantage of use of the patented super luer-lock and microcannulas system for use with the closed-syringe system. A sequential explanation of equipment selection for minimally traumatic lipoaspiration in small volumes is presented, including use of blunt injection cannulas to reduce risk of embolism.

Results: Thousands of AFG have proven safe and efficacious for lipoaspiration techniques for large and small structural fat grafting procedures. The importance and advantages of gentle harvesting of the adipose tissue complex has become very clear in the past 5 years. The closedsyringe system offers a minimally invasive, gentle system with which to mobilize subdermal fat tissues in a suspension form. Resulting total nuclear counting of undifferentiated cells of the adipose-derived -SVF suggests that the yield achieved is better than use of always-on, constant mechanical pump applied vacuum systems.

Conclusion: Use of a closed-syringe lipoaspiration system featuring disposable microcannulas offers a safe and effective means of harvesting small volumes of nonmanipulated adipose tissues and its accompanying progenitor cells within the SVF. Closed syringes and microcannulas are available as safe, sterile, disposable, compact systems for acquiring high-quality AFG. Presented is a detailed, step-by-step, proven protocol for performing quality autologous structural adipose transplantation.

Keywords: autologous fat grafting, closed syringe lipoaspiration, adipose-derived adult stemstromal cell, bioscaffolds, lipoaspiration/liposuction, stromal vascular fraction (SVF)

\section{Introduction and background}

For many years, cosmetic plastic surgeons have recognized the value of low-pressure lipoaspiration for successful transplantation of adipose tissue for structural augmentation. In the introductory years (1980-1990) of liposuction techniques, autologous fat grafting (AFG) was considered unpredictable. Once bioengineers discovered the actual mechanisms by which lipoaspiration worked, the closed syringe system for gentle harvesting and transplantation was developed and patented. Early belief that effective lipoaspiration was directly related to force of vacuum was replaced by understanding, that, introduction of fluid into the fat layers permitted the adipocyte cells and stroma elements to enter into
Correspondence: Robert W Alexander Department of Surgery, School of Medicine and Dentistry, University of Washington, Seattle, WA, USA

Tel +l 4067774477

Fax +l 8667665458

Email rwamd@cybernetl.com 
suspension. This suspension was easily extracted through use of closed syringes, and provided adipose tissues with reduced damage and improved grafting results. ${ }^{1}$

In the mid-1980s, the importance of tumescent fluid distribution was first appreciated, and more value was placed on extensive pre-tunneling (moving cannula without applying vacuum). This technique better distributes local solution and enhances the ability to mobilize the adipose tissues into a suspension state, which yields more successful and predictable autologous fat grafts (AFG). ${ }^{1}$

In the early $2000 \mathrm{~s}$, appreciation of the potential of adipose tissue and its related stromal elements led to examination of the adipose-derived adult mesenchymal stem cell content (AD-MSC). ${ }^{2,3}$ Evidence has clearly shown the key importance of the progenitor cells (SVF) and extracellular matrix (ECM) as integral contributors to the tissue maintenance and healing processes. ${ }^{4}$ Studies of adipocyte replenishment (following normal senescence and cellular death) showed that these attached progenitor cells were activated to form adipocytes and thereby maintain adipose tissue integrity over time. ${ }^{5,6}$ Since there is easy accessibility and greater availability of multipotent progenitor cells for all mesogenic lines within adipose tissues, utilization of AD-MSC/SVF has become a central focus in optimizing effectiveness of autologous fat acquisition and grafting in cosmetic plastic surgery and clinical regenerative medicine..$^{7-10}$

Preclinical and clinical applications have been reported in many scientific studies in the biological, bioengineering, and clinical medical literature. ${ }^{11}$ Cosmetic plastic surgeons initially focused on understanding the mechanisms to achieve safe and effective AFG. It was believed that intact cellular (mature adipocytes) transplantation was the most important goal. ${ }^{12}$ However, it has become crystal clear that mature adult adipocytes transplanted may be the least important feature producing long-term success, even in structural fat augmentation graft applications. Current beliefs are that success in long-term AFG is actually due to activation of adherent progenitor cells (attached to mature adipocytes, ECM and SVF), and proliferation of those progenitor cells to differentiate into the target cell for replacement. ${ }^{4,13-15}$

As an example, placement of lipoaspirants into existing adipose tissue favors proliferation and differentiation into adipose cell phenotypes. As understanding of the maintenance (homeostatic) and replenishment of adipose cell cycles in vivo increases, extensive research has been devoted to the study of microenvironment (niche), cell-tocell/cell-to-matrix factors, and autocrine/paracrine signaling system functions. Since AD-MSC/SVF are capable of differentiation for all mesogenic lines, including: 1) chondrogenic; 2) fibro-muscular (including tendon, ligament, and skeletal and cardiac muscle); 3) osteogenic; and 4) adipogenic cell lines, uses in clinical applications have increased. ${ }^{16-22}$

Rapidly accumulating clinical data on the safety and efficacy of AD-MSC/SVF in vivo provides clear evidence that adipose tissue grafts possess extensive potentials in wound healing, well beyond the structural augmentation in cosmetic plastic surgical uses.

Understanding these mechanisms results in important application potentials for aesthetic, reconstructive, and regenerative medicine. Reporting of preclinical, early clinical, and controlled studies in animal and human models shows worldwide recognition of the potential uses of these cells in diverse areas of medicine and surgery. ${ }^{23-25}$

\section{AFG as heterogeneous stromal-stem cell source and living bioscaffolding for use in aesthetic reconstructive surgery}

Research and clinical applications have led to appreciation of the existence of a large heterogeneous, undifferentiated nucleated cell populations and extensive native bioactive scaffolding that is an integral component of the total SVF components within the adipose tissues. ${ }^{4}$

Most reports examining the nature and components of the stromal vascular fraction (SVF) have come from decades of study on bone marrow-derived stem cells (BM-SCs). ${ }^{26} \mathrm{It}$ is now recognized that AD-MSCs have essentially the same capabilities while offering significant advantages over BMMSCs utilization. These include: 1) very similar progenitor differentiation capabilities; 2) important cellular subsets (such as mesenchymal and perivascular cells) are found in much higher concentrations compared to bone marrow aspirants $(>1500 \mathrm{X})$; 3 ) less expensive to harvest; 4) less invasive (safer); 5) readily available tissue; and 6) less technically demanding than bone marrow penetration and cellular harvest. These features have led to increased uses in many areas of medicine and surgery. ${ }^{27-33}$

With identification of the near-terminally differentiated cells (pre-adipocytes) attached directly to the cytoplastic surface of all mature adipocytes by Granneman et al, homeostatic mechanisms with adipose metabolism have been better understood. ${ }^{34}$ With advanced understanding of the processes of homeostatic adipose replacement, many reasons why carefully harvested autologous fat grafts for structural augmentation is effective have become much more predictable and understood than in years past. ${ }^{35-37}$

Technology is now available to effectively isolate/concentrate these progenitor cells, but such manipulation involving 
chemical digestion is not permitted for clinical use within the US. Therefore, utilization of non-manipulated AFG/ PRP concentrates remains the only option at this time. With recognition that lipoaspiration (versus en bloc excised fat) acquires a bit over $50 \%$ of the native multipotent, nucleated cell populations, such isolation and concentration protocols have gained favor in efforts to help restore the multipotent cell numbers to near native levels. ${ }^{38}$ At this time, such digestion and isolation procedures are prohibited by the regulatory agencies within the US, as they are considered more-than-minimally manipulated tissues and subject to such rules.

Laboratory findings suggest that use of closed-syringe harvesting techniques produces higher nucleated cellular counts as compared with low-pressure wall suction or detuned lipoaspiration mechanical pumps (Alexander RW, Mandle R; Harvard Biosciences Laboratory, unpublished data, 2012) (See Figure 1).

The patented Tulip ${ }^{\mathrm{TM}}$ closed-syringe system (Tulip Medical Systems, San Diego, CA, USA), with its array of microcannulas (small volumes- $<100 \mathrm{cc}$ ) and standard volume reduction cannulas (for $>100$ cc liporeduction and contouring), is a recognized and proven lipoaspiration system. Explanation and detailed discussion of a repeatable, effective, and safe protocol for adipose tissue complex harvest for cosmetic plastic surgeons will be provided in this paper.

\section{Materials and methods}

\section{Selection of lipoaspiration sites}

The lower abdomen and flank areas of both males and females are considered ideal adipose donor sites due to distribution of human adipose tissues and relatively large deposits. Choice of aspiration sites in the medial and lateral thigh/buttocks areas is sometimes favored for lipoaspiration and adipose graft harvesting in female patients due to genetic distribution within the gynoid body type. In patients with very low percentage body fat needing autologous grafts, use of a high-definition ultrasound probe is helpful to determine the thickness and depth of adipose deposits that can be acquired.

\section{Preparation of lipoaspiration sites (donor and recipient)}

The patients may be placed in either supine or lateral decubitus position to facilitate the preparation and complete sterile isolation of the proposed donor area(s). It is considered important to follow a standard sterile protocol for both the harvesting and placement sites. Routine operative site asepsis should be maintained in all cases, with patients marked in an
Cell counts per $\mathrm{mL}$ sample

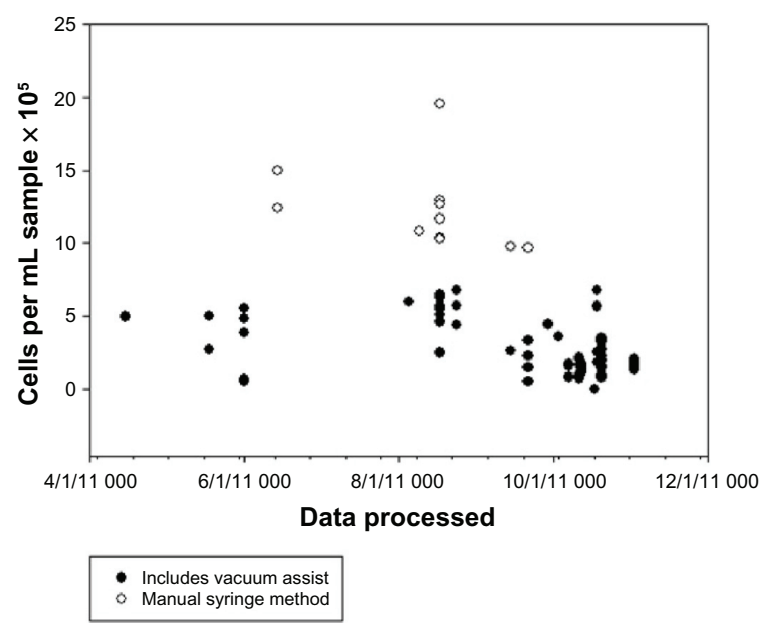

Figure I Comparison of syringe-harvested isolated adipose-derived mesenchymal stromal cell (AD-MSC) counts (open circles) versus use of low-pressure machine harvest (wall suction or detuned lipoaspiration machine [dark circles]).

Notes: Vertical axis shows AD-MSCs per cc. Microcannula lipoaspirants were harvested by offset $2.1 \mathrm{~mm}$ OD cannulas and processed by collagenase digestion to isolate cellular subsets; counts were based on MSC-specific cell-marker characterization.

Abbreviation: OD, outside diameter.

upright or standing position to effectively mark the area of available or unwanted adipose tissue deposits.

\section{Microcannula instrumentation}

The patented Tulip ${ }^{\mathrm{TM}}$ closed-syringe system for lipoaspiration features very smooth cannulas and a "super" luer-lock connection for use with standard luer-lock syringes (Figure 2). This hub connection is a very important component of the closed-syringe and microcannula system, in that it provides an excellent seal for maintaining even vacuum forces desirable during lipoaspiration (Figure 3). Since the super-luer lock connection seals at both the internal luer connection and at the outer ring of the standard luer connection, it thereby provides a very stable, rigid base. Using microcannula sized instruments are very flexible compared to standard cannula

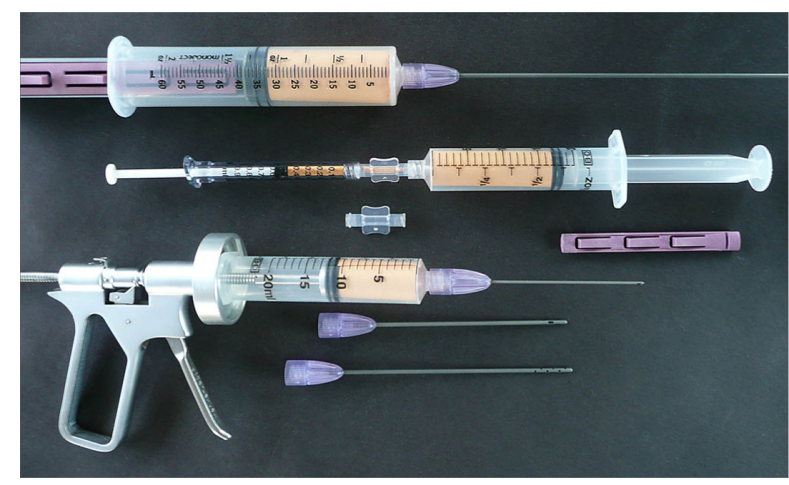

Figure 2 Complete closed-syringe microcannula system (Tulip ${ }^{\circledR}$ GEMS $^{\mathrm{TM}}$, Tulip Medical Systems, San Diego, CA, USA). 
types. The super luer lok provides substantial stability and control in these cases. Internal female luer-type connectors on some microcannula systems on the market become less efficient when cannulas are redirected, placing a torque on the junction of cannula-syringe barrel, and allowing air leakage into the closed system (particularly in the longer cannula selections). This does not usually prevent aspiration capabilities, but it does decrease the efficiency and may introduce cavitation to the harvest tissues.

Two standard options for microcannula selection offered within the Tulip ${ }^{\mathrm{TM}}$ system are:

1. Cell-friendly microcannula option (autoclavable) (Figure 4). These cannulas are internally polished by microabrasive extrusion process to maximize internal smoothness and reduce adipose tissue damage to the adipocytes, precursor cells, and their accompanying matrix. External cannula anodizing processes provide a smoother surface for ease of passage within the subdermal adipose plane. This is a popular design utilized by cosmetic plastic surgeons for liporeduction as well as harvesting of AFG for structural augmentation procedures. References the closed syringe system instruments are also available for larger volume liporeduction (liposuction) cases and in large volume structural graft cases. In cannulas of less than $3.0 \mathrm{~mm}$, it is important to thoroughly flush with water-prep soap mix, followed by ultrasonic cleaning, and thorough re-flushing with water prior to steam or gas sterilization.

2. Sterile, coated disposable microcannula option (Figure 5). Use of disposable microcannulas in small diameters of

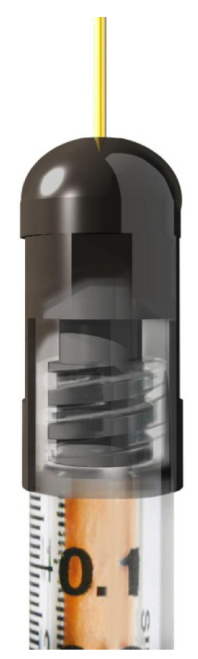

Figure 3 Tulip $^{\circledast}$ super luer-lock connection (Tulip Medical Systems, San Diego, CA, USA).

Note: Seal on threaded standard luer connection (internal) plus seal on outside of standard luer external hub.

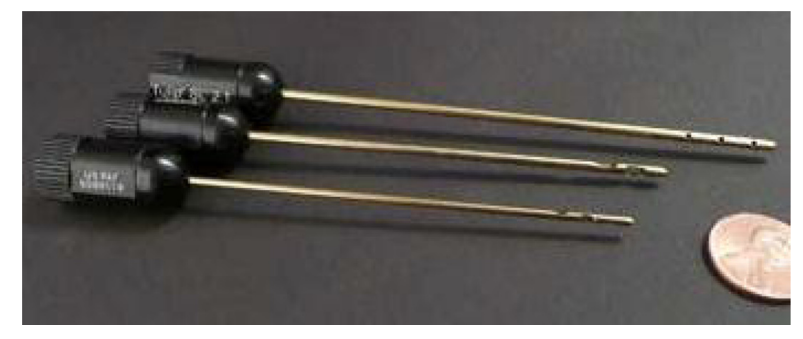

Figure 4 Tulip $^{\circledR}$ (Tulip Medical Systems, San Diego, CA, USA) cell-friendly (autoclavable) microcannulas.

less than $3.0 \mathrm{~mm}$ (range $0.9-2.4 \mathrm{~mm}$ outside diameter [OD]) presents a significant challenge to ensure proper and effective cleaning-sterilization cycles. This makes a disposable option attractive, particularly in the smaller diameter cannula group. These are packaged and labeled in a sterile wrap, and can be opened directly onto the sterile field or back table. Featuring the super luer-lock base, these stainless steel cannulas are totally coated internally and externally with a hydrophilic material that provides an extremely smooth coating, which passes through adipose tissues with minimal resistance and trauma. Initially, hydrogel coatings were applied on both internal and external surfaces, but now have been replaced by more efficient and effective coating materials, featuring more than 20 times more lubriciousness than previous coating materials. It is believed that the least cellular and tissue trauma created, the better the quality of the adipose grafts.

With increased recognition of difficulties in effectively cleaning the nondisposable microcannulas $(<3.0 \mathrm{~mm})$, most surgeons and operating facilities are choosing use of completely disposable infiltration, harvesting, and injection cannulas.

\section{Selection of microcannula length and diameters}

For small volume applications ( $<100 \mathrm{cc})$, it is recommended to use a small, multiport infiltrator cannula for even and thorough distribution of local anesthesia throughout the adipose donor layer. Openings near the tip are multiple and oriented for 360-degree distribution of the tumescent local solution while moving through the subdermal fat layers. It is common for practitioners to use this infiltration cannula in diameters of 2.1-2.4 mm OD and a length of 10-20 cm (Figure 6 [top]).

Harvesting cannulas are designed to actually acquire the adipose tissue grafts from the subdermal fat plane, following the same pattern and location of local anesthesia distribution (Figure 6 [middle]). 


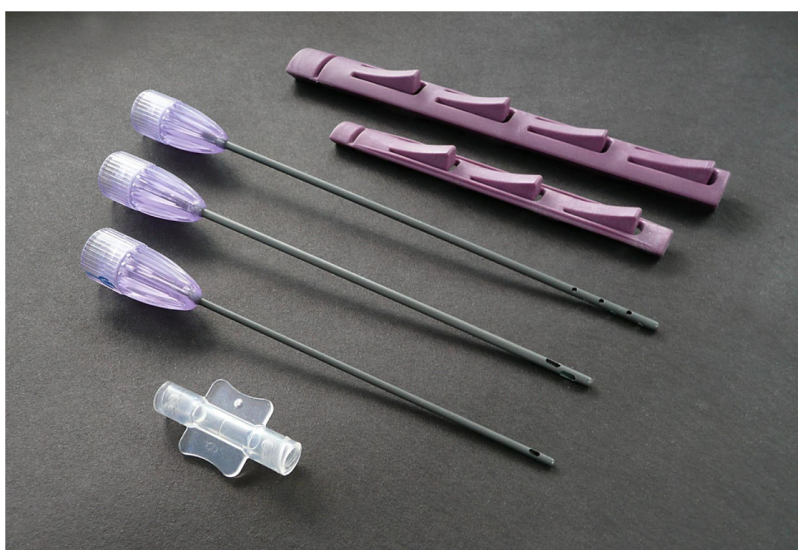

Figure 5 Disposable microcannula cannulas for closed-syringe lipoaspiration of small-volume autologous adipose grafting (Tulip ${ }^{\circledR}$ GEMS TM, Tulip Medical Systems, San Diego, CA, USA).

Notes: Top: snap-lock option for syringes; middle: coated multiport infiltrator, offset harvester, single-port injector; bottom: clear luer-luer anaerobic transfer.

The openings on the harvesting cannulas are usually provided as inline or offset openings (meaning in a nonlinear pattern near the tip of the cannula). These vary in diameter from $1.67 \mathrm{~mm}$ to $2.4 \mathrm{~mm}(\mathrm{OD})$, with a length of $10-20 \mathrm{~cm}$. Selection of a slightly shorter harvesting cannula compared to length of infiltrator makes it somewhat easier to remain within the local anesthesia distribution areas in awake patients.

Closed-system syringe locks are available in two options, an "external" and an "internal" form. The external locks are specifically designed for use on Bectin Dickinson Co. (Franklin Lakes, NJ, USA) or Monoject (CovidienKendall, Mansfield, MA, USA) 10/12 and 20 cc luer-lock syringes and $60 \mathrm{cc}$ Toomey tip syringes to hold the syringe plunger in a fully drawn position during the application of vacuum. Before application of vacuum by pulling the syringe plunger to the desired level, it is essential to draw sterile saline fluid into the cannula to completely displace all air within the system. When pulled and twisted into the locked position, the edge of the lock engages the side of the plunger permitting the physician to apply even and gentle vacuum pressure while moving the cannula through the tumesced adipose layer. The internal type lock, called a snap lock, universally fits a range of different syringe sizes from various manufacturers. (Figure 7).

Anaerobic transfers (luer-to-luer) are utilized to facilitate loading of treatment syringes prior to grafting procedures, and for optional use of additives to the grafts (such as combining platelet concentrates [HD PRP] and adipose grafts into one syringe [Figure 5 (bottom)]). They are also useful for transferring the treatment mix into syringe sizes of physician preference for injection and avoiding

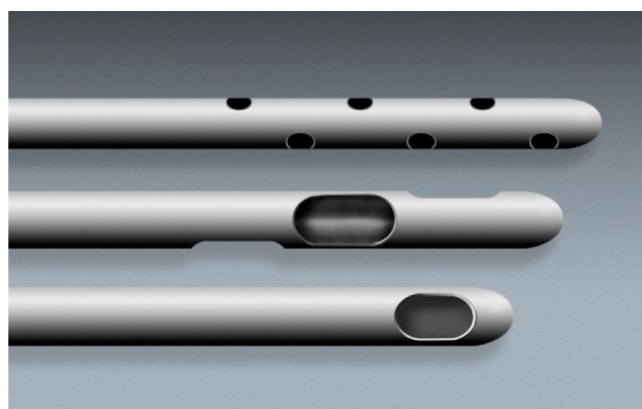

Figure 6 Close-up of microcannula openings.

Notes: Top: multiport infiltrator cannula; middle: offset harvester cannula; bottom: single-port injector cannula.

undesirable exposure of the harvested graft to air (Figure 8). It is considered important to avoid excessive air exposure to grafts due to the potential for contamination by airborne particles or pathogens. Techniques described as helpful in free lipid removal (such as Telfa ${ }^{\mathrm{TM}}$ "rolling"; Johnson \& Johnson, New Brunswick, NJ, USA) are vulnerable to such contamination.

A controlled aliquot injector gun is useful for placement of controlled $0.5 \mathrm{cc}$ aliquots of graft into the prepared tunnels and locations. When additives are added to the adipose tissue graft, the density of the injection material may be increased (eg, use of HD PRP plus thrombin to provide rapid release of platelet-derived growth factors and signal proteins). This may result in the physician requiring more force to inject into the tissue site, or, to encounter sudden and uneven distribution of desired small aliquots of graft with prepared tunnels associated with adipose matrix density within the graft itself. Single-trigger pull provides exact volumes of solution to be placed with less pressure required by the provider (Figure 9).

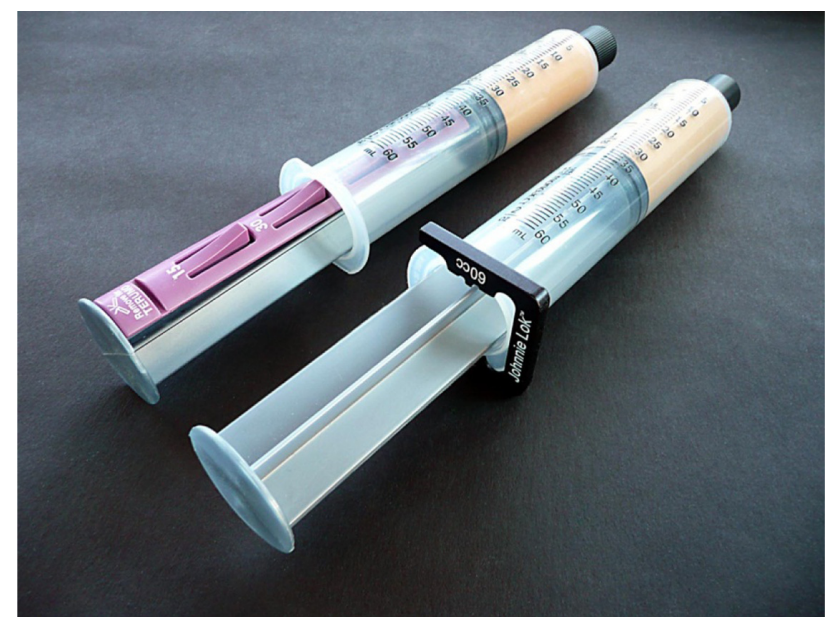

Figure 7 Closed-syringe lock options.

Notes: Left: internal snap lock (disposable); right: external Johnnie Lok ${ }^{\mathrm{TM}}$ (reusable) Tulip ${ }^{\circledR}$ (Tulip Medical Systems, San Diego, CA, USA). 


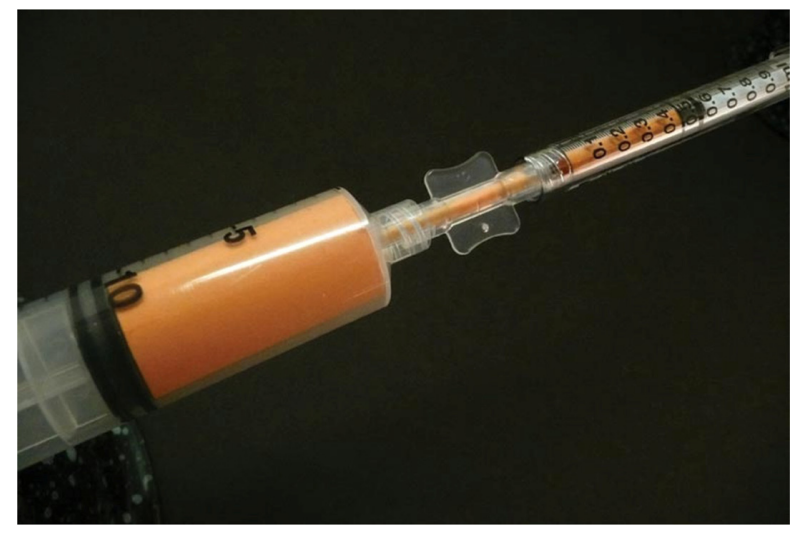

Figure 8 Anaerobic luer-to-luer transfer shown loading adipose graft to I cc treatment syringe.

\section{Recommended step-by-step protocol for performing closed system microcannula lipoaspiration}

It is recommended that the area of donor and recipient sites be outlined with the patient in upright position and using a skin-marking pencil. This area will then be prepped, draped, and isolated to expose the thickest deposit of palpable fat tissues, and serve as a distribution pattern for local anesthetic infiltration (see Figure 10 for an example).

After marking, preparation, and sterile isolation of the donor area, an 18-20 $\mathrm{g}$ beveled needle is held such that the side edge is held vertical to the skin. This side edge is utilized to create a small, vertical (slit-like) opening, extending through the epidermis and dermis into the subdermal fat plane of the donor site. It is very important to avoid too large an opening, as closed-syringe-system vacuum depends on maintaining a tight side wall opening to ensure even vacuum application. Use of stab incisions with scalpel blades of \#15 or \#11 sizes tends to create larger than necessary or desired openings. The opening is made larger by selectively cutting the dermal layer (under the skin surface) with the edge of the needle bevel. In large cannula sizes, use of a tapered stainless sharp trocar $(3 \mathrm{~mm})$ is utilized to permit a snug fit of the aspiration cannulas into the desired space for cannulas of $>3 \mathrm{~mm}$ OD. This allows the introduction of the multiport infiltration cannula through the skin and the subdermal fascia (and in the anterior abdominal wall example, should perforate and remain beneath Scarpa's fascia) (Figure 11).

Following entry, the multiport infiltrator cannula should be passed in a horizontal fashion within deeper aspects of the subcutaneous donor fat deposit, above the muscular layer, in a "spokes-of-a-wheel" pattern. Pinching the skin-fat tissues may help in passing the cannula. During movement of the infiltrating cannula, very slow injection of tumescent local anesthesia fluid, during both entry and withdrawal strokes improves even distribution. Placement in layers, beginning in the deeper aspects, and moving to more superficial levels is most effective. The importance of avoiding "pooling" of the tumescent local anesthesia is that evenly distributing liquids improves the efficiency of harvest by providing a suspensory fluid carrier for the adipose graft tissues, as well as excellent patient comfort. If tumescent fluids are rapidly injected or injected only in a few locations within the fat deposits, pooling is often the result. This pooling is recognized by the surgeon by the presence of larger amounts of infranatant fluids seen after gravity decantation or centrifugation.

In typical small volume grafting cases, use of tumescent local anesthesia in a range of $20-30 \mathrm{cc}$ is common. As a general guideline, it is important to place at least $1 \mathrm{cc}$ of tumescent local per $1 \mathrm{cc}$ of anticipated graft to be harvested. For example, if the plan is to aspirate $50 \mathrm{cc}$ of adipose tissues, then use of $50 \mathrm{cc}$ or greater fluid volume is distributed with the adipose layer to provide the fluid carrier for extraction of the grafts. A common example of the component of tumescent solution is to add the contents of a $50 \mathrm{cc}$ multidose vial of local anesthetic (eg, $0.5 \%$ to $1.0 \%$ xylocaine with or without epinephrine $[1: 100,000])$ to $1 \mathrm{~L}$ of sterile saline or balanced salt solution to provide sufficient tumescent fluid for lipoaspiration.

Upon completion of even distribution of tumescent fluid within the proposed donor area, it is recommended that repetitive passage of the infiltrating cannula throughout the tumesced donor area (termed "pre-tunneling") multiple times is important and very helpful to attain an even and high quality graft. This more thoroughly distributes local anesthetic fluid for patient comfort, and it also provides the needed "carrier fluid" to effectively suspend the adipose tissues prior to harvesting with low pressure and minimal bleeding. This is a very important step that will improve comfort during harvest, plus make extraction more efficient and result in markedly less volume of the unwanted infranatant fluid layer.

In small-volume transfers, most practitioners select a $20 \mathrm{cc}$ luer syringe attached to the harvesting microcannula with a mounted locking device. A very small volume (1-2 cc) of sterile $0.9 \%$ saline is drawn into the cannula to displace air from the system prior to insertion into the harvest (donor site). This is termed "charging" the syringe device, and is necessary to eliminate all air from within the cannula and syringe, thereby avoiding cavitation produced when using mechanical pump suction devices (wall suction, detuned lipoaspiration machines, etc). 
Once the harvesting cannula is inserted into the locally tumesced and pre-tunneled adipose layer, the syringe plunger is drawn to partial or full extension depending on desired vacuum pressure, and twisted to provide a "lock," if using an external type, or a ledge, which snaps to hold the plunger in one of three positions. After application of vacuum, the physician is free to move the harvesting cannula in a forward and back series of passages. It is important that these passages are within the same plane and of the same pattern used during the placement of tumescent solution. Adipose return, at first, will be somewhat slower, as the graft tissue must be in suspension to be able to be easily extracted. Continuing these movements with vacuum applied will yield adipose tissues with minimal bleeding in most patients.

During the displacement of air, it is recommended that 4 inch by 4 inch sterile gauze should be held over the harvesting tip openings to avoid spraying contents. Occasionally, in cases where there is a larger volume of infranatant fluid (the layer immediately below the fat tissues), simply express the liquid portion, reinsert the harvesting cannula into the harvest site, lock the plunger, and gather more graft tissue. In the event of vacuum pressure loss during the harvesting process, it is sometimes necessary to completely remove the harvesting cannula from the donor site, carefully expressing all air from within the cannula. When this is completed, reinsertion of the cannula is performed, and vacuum is re-established by withdrawing (pulling) the plunger and re-locking it.

The yellow adipose grafts will quickly gravity separate from the underlying (infranatant) fluid, resulting in the graft floating on top of the small fluid volume within the syringe system. Test tube/syringe stands or decanting stands are available to facilitate this initial gravity separation (Figure 12A and B).

Remember that a common cause of increased infranatant volume in the decanted syringe is inadequate distribution of local fluid, creating a pooling effect, which reduces efficiency of adipose harvest. It is for this reason that extensive pre-tunneling is highly recommended prior to application of any vacuum to the tissues. Upon completion of aspiration of the desired graft volume, the harvester cannula is removed, and the syringe endcapped and placed in a vertical position in a standard test tube rack or directly mounted on a luer base of a decanting stand to allow gravity to separate three distinct layers within the harvesting syringe. This usually requires decantation for approximately 2-3 minutes or centrifugation (discussed below) at $1000 \mathrm{~g}$ for 3-4 minutes. After layer separation, practitioners expel the unwanted liquid layer (infranatant liquid) on which the fat graft floats, which is expressed into sterile containers for disposal.

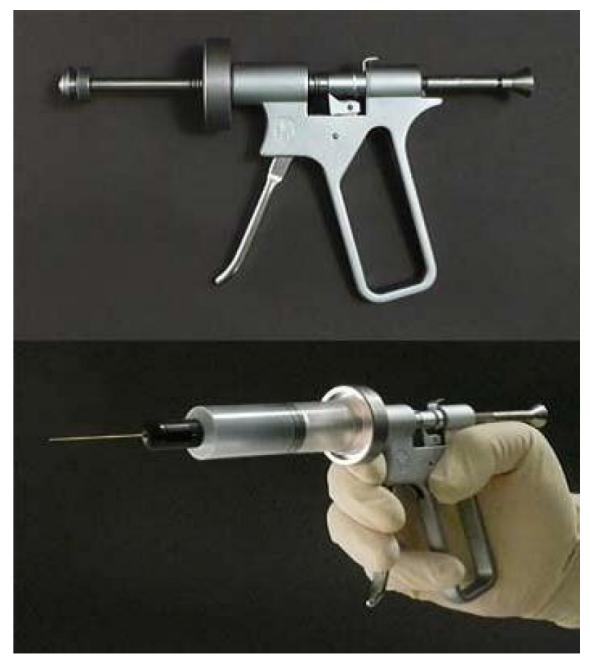

Figure 9 Mechanical injector gun (Tulip ${ }^{\circledR}$, Tulip Medical Systems, San Diego, CA, USA).

Following removal of the infranatant fluid layer, if additional graft is needed, it is possible to reinsert the harvester into the donor site to acquire more graft tissue (adipocytes and adipose-derived tissue stromal vascular fraction), additional decanting/centrifugation, followed by anaerobic transfer loading of the graft into the treatment syringes of choice.

When the desired volume is attained, lipoaspiration is completed, and the small opening is covered with a triple antibiotic ointment and a Coverlet (BSN Medical, Charlotte, NC, USA) bandage (adhesive on all sides).

In the event of loss of vacuum (evidenced by a hissing sound), the cannula is simply removed from the adipose tissues, fluid advanced into the harvesting cannula to eliminate air from the system, cannula reinserted, and plunger pulled open and locked to restart the aspiration process.

Ideally, the more thorough removal of unwanted fluids from the graft yields a more dense cellular graft. It is important to effectively avoid the top layer (free lipid layer) from being included in the final AFG transplantation. In addition, the free lipid layer (clear yellow liquid above the harvested graft) should be avoided, as it is an irritant and prolongs the healing of the graft tissues in that it must be removed during the process by macrophages, etc, over time.

\section{Centrifugation option in AFG (recommended)}

The desire to produce the best compact grafts with minimal remaining fluid excess has led many to include use of optimal centrifugation (1000 $g$ for 3-4 minutes) to effectively compress the graft materials, help to more precisely separate fluid from harvested graft, and facilitate removal of the unwanted free lipid component (Figure 13) 39 "Several providers of 


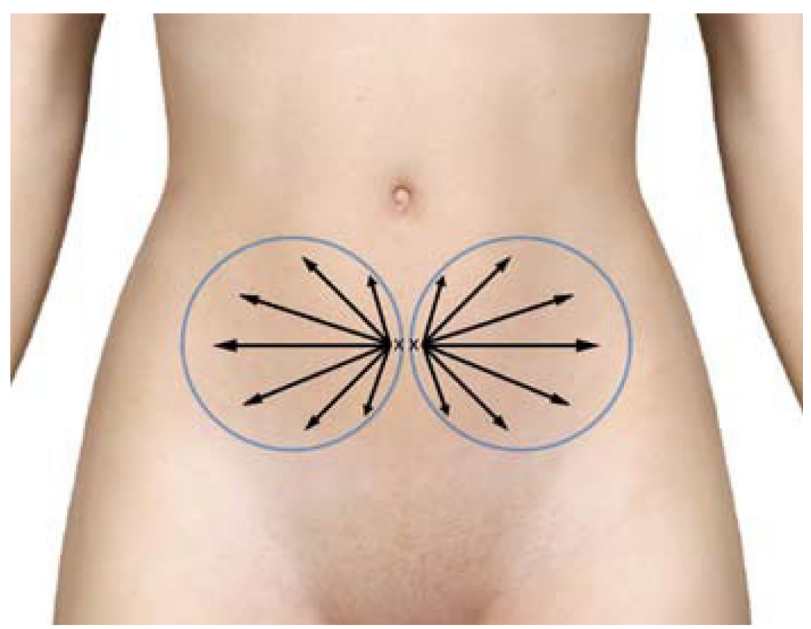

Figure 10 Example marking of lower abdomen site for harvesting of subdermal adipose tissue.

Note: Arrows show wheel-spoke pattern of tumescent infiltration and harvesting of abdominal donor site.

tissue processing syringes offer the ability to more easily remove the lipid layer from the graft tissues desired (see Figure 14). Whether by disk or weighted plunger, the free lipids are allowed to pass out of the upper graft tissues and remain in the harvesting syringe itself. The ability to thoroughly remove unnecessary fluid from the graft provides the surgeon an opportunity to avoid the mandatory "overcorrection" needed due to the amount of remaining carrier fluids within the graft. Further, use of a disposable separator disk, within the centrifuge processing syringe, ensures more complete separation of the free lipid free lipid layer. Its removal prevents the resulting irritation and inflammatory response when removing the unwanted additional free lipid from within a recipient site (Figure 14).

Some practitioners perform one to two rinses with sterile saline to help reduce any residual local anesthetic solution and red blood cells. ${ }^{40}$

After decantation and/or centrifugation steps, the graft preparation is ready for placement into treatment syringes of physician's choice. It is important to use clear anaerobic transfers (luer-to-luer connectors) to load the individual application syringes from the prepared, compressed graft

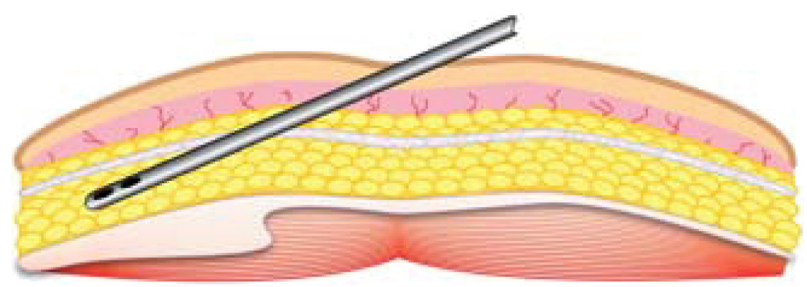

Figure II Placement of microcannula below Scarpa's fascia in the adipose tissue plane.
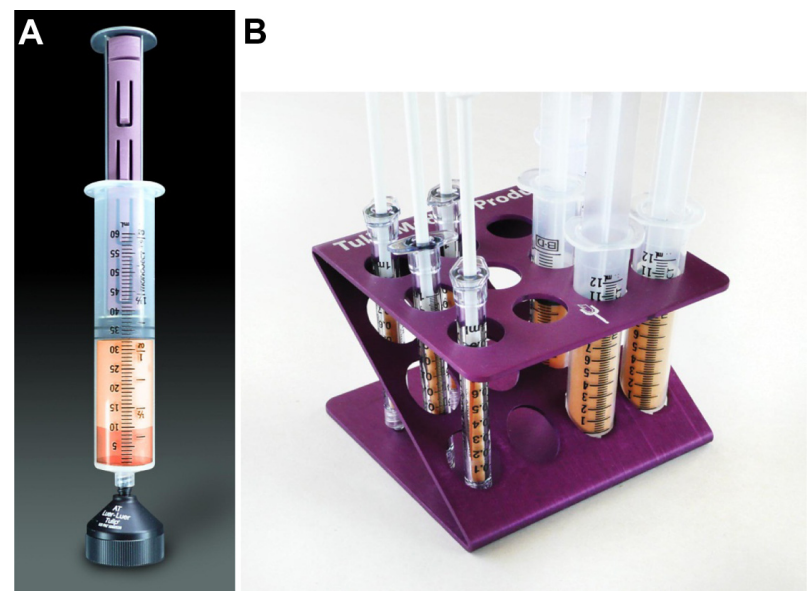

Figure 12 (A) Gravity decant base (luer); (B) gravity decant in test tube rack.

(Figure 15). They are advantageous in that they prevent external air exposure and potential for contamination and permit use of additives, such as high-density platelet-rich plasma (HD PRP) concentrates. Use of HD PRP concentrates are believed to further enhance the healing, graft acceptance, and provide high concentrations of helpful growth factors and signal proteins.

Treatment-standard luer syringes are then mounted with the desired injection cannulas, using coated, single-port cannulas or the surgeons, preferred injector needles. Use of blunt-tip, coated cannulas is recommended, particularly within the facial recipient areas to lower the risk of embolism caused by inadvertent injection of the adipose graft intravascularly. The injection cannulas are available in a variety of lengths and diameters (ranging from

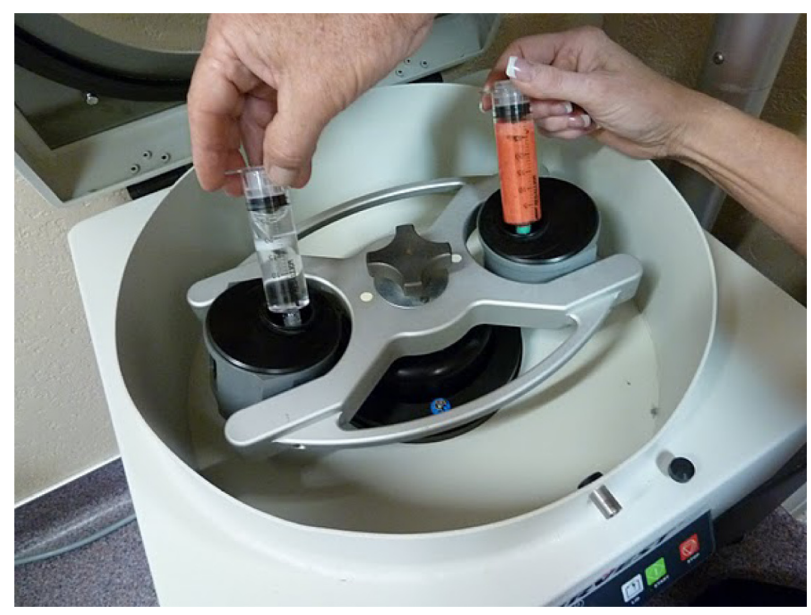

Figure 13 The SmartPRep ${ }^{\circledR 2}$ APC ${ }^{+T M}$ centrifuge, which forms part of the AdiPRep ${ }^{\mathrm{TM}}$ Adipose Transfer System (Harvest-Terumo, Plymouth, MA, USA).

Note: The left syringe is the counterbalance (containing saline) and the right contains the harvested adipose graft in a disposable processing syringe (with disk) prior to centrifugation ( $1000 \mathrm{~g}$ for 4 minutes). 
$0.9 \mathrm{~mm}$ to $1.47 \mathrm{~mm} \mathrm{OD})$, to accommodate the surgeon's preference and specific areas to be grafted. Some elect to inject with sharp needles ranging in size from $18 \mathrm{~g}$ to $25 \mathrm{~g}$ (Figure 16).

The typical graft recipient bed is prepared and developed by pre-tunneling to create a "potential" space, which is subsequently filled in small aliquots and in layers as the injection cannula is being withdrawn.

It is recommended that the donor sites be dressed in a proper fashion. Use of small, sterile absorbing gauze dressing placed over the skin opening created to place the tumescent fluids into the subdermal tissues. Further, use of compression and closed cell, medical grade foam (TenderFoam ${ }^{\text {TM}}$; T\&N Industries, San Diego, CA, USA) will eliminate or minimize post-harvest bruising of the donor area. It is believed that use of foam and compression reduces the lymphatic dispersion of small amounts of red blood cells or breakdown pigments remaining within the surgical harvest site in the form of bruising or discoloration. Firm compression of the gauze and TenderFoam for 24-48 hours is typically effective (Figure 17A and B).

\section{Discussion}

When the mechanisms involved in liposuction technologies were recognized in the mid-late 1980s, the ability to provide small- and large-volume liposuction via the closed-syringe system was proven safe and more predictable and effective. By use of low vacuum pressure and avoiding air within the system, the closed syringe system improved the results of

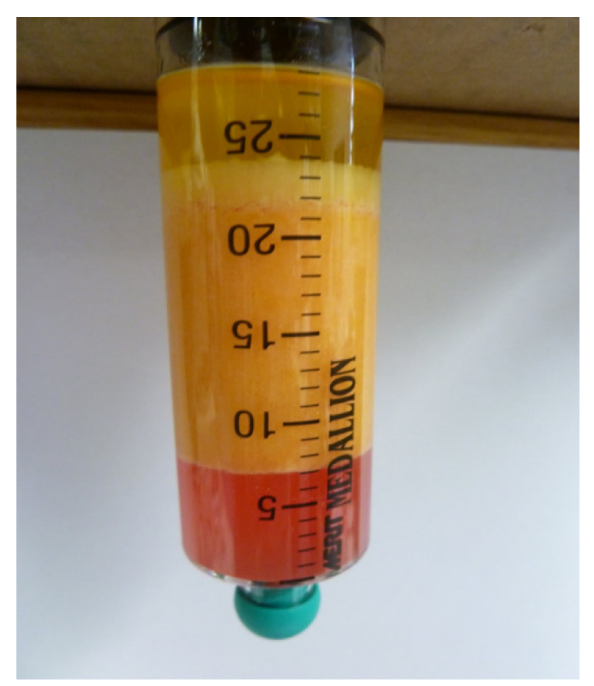

Figure I 4 Close-up of a disposable processing syringe containing extracted tissue that has separated.

Notes: The top layer is free lipid in and above the white separator disk, the middle layer is compressed adipose tissue graft, and the bottom layer is infranatant fluid and debris. liporeduction surgery and autologous fat grafting success. This included removal of significantly larger volumes in a single session and reduction of deposits within the superficial plane. The ability to safely and effectively reduce unwanted fat deposits, coupled with improved skin redraping resulted in improved surgical outcomes. Besides volume implications, closed-syringe lipoaspiration launched the beginning of more consistent and predictable autologous fat grafting procedures, with improved safety, efficacy, and predictability within aesthetic surgical applications. Structural fat grafting, utilizing the exact techniques described herein, has been completed many thousands of times by many cosmetic plastic surgeons (Figure 18A-J).

Scientific research has now provided important information to help explain the homeostatic and transplant acceptance mechanisms accomplished by autologous fat. As appreciation of the biocellular nature of the adipose tissue complex increases, the importance and value of the SVF has gained major attention. ${ }^{4}$

The authors believe that high-quality, dense cellular grafts are very important to the effectiveness of structural fat grafting procedures. To that end, ensurance of complete layer separation of the harvested grafts provided by optimized centrifugation is considered advantageous. In addition to use of such centrifugation, the use of a disposable processing syringe (with separator disk) further ensures that free lipids are effectively removed during graft preparation. The AdiPRep ${ }^{\mathrm{TM}}$ system (Harvest-Terumo, Plymouth, MA, USA) provides such a system using the same centrifuge as used for producing HD PRP concentrates (SmartPRep IITM; HarvestTerumo, Plymouth, MA, USA). Use of closed systems

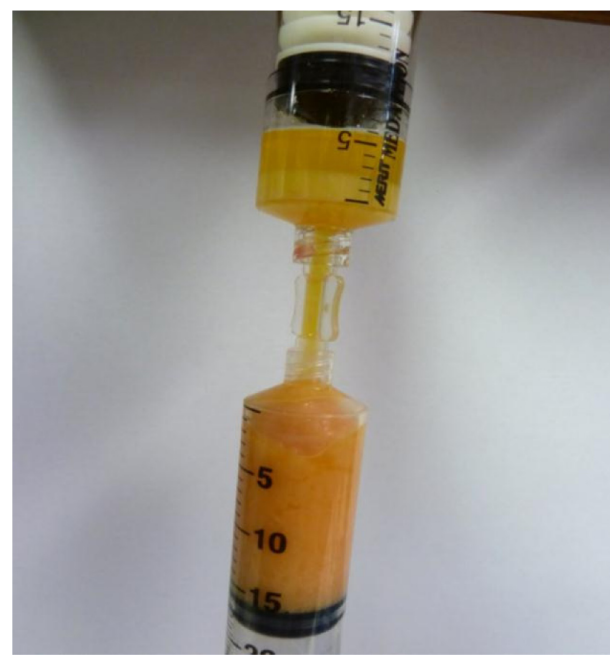

Figure 15 Anaerobic transfer from disposable processing syringe (above) to adipose fat graft syringe (below). 


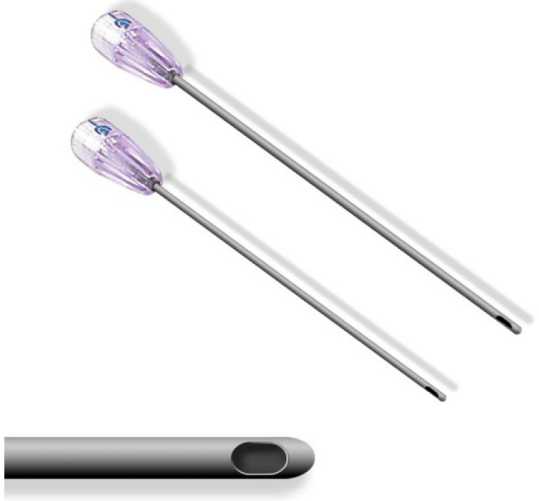

Figure 16 Tulip $^{\circledR}$ GEMS $^{\text {TM }}$ (Tulip Medical Systems, San Diego, CA, USA) single-port injection cannulas (top) and close-up of the tip of one of these (bottom).

for the harvesting and preparation of high-quality grafts is considered of great importance.

It is becoming mainstream knowledge that the actual transplanted mature adipocytes are gradually lost, while serving an important role in their own replacement from attached near-terminally differentiated cells (pre-adipocytes). For several years, leading practitioners sought to achieve pure, adipocyte grafts, without regard to the SVF components

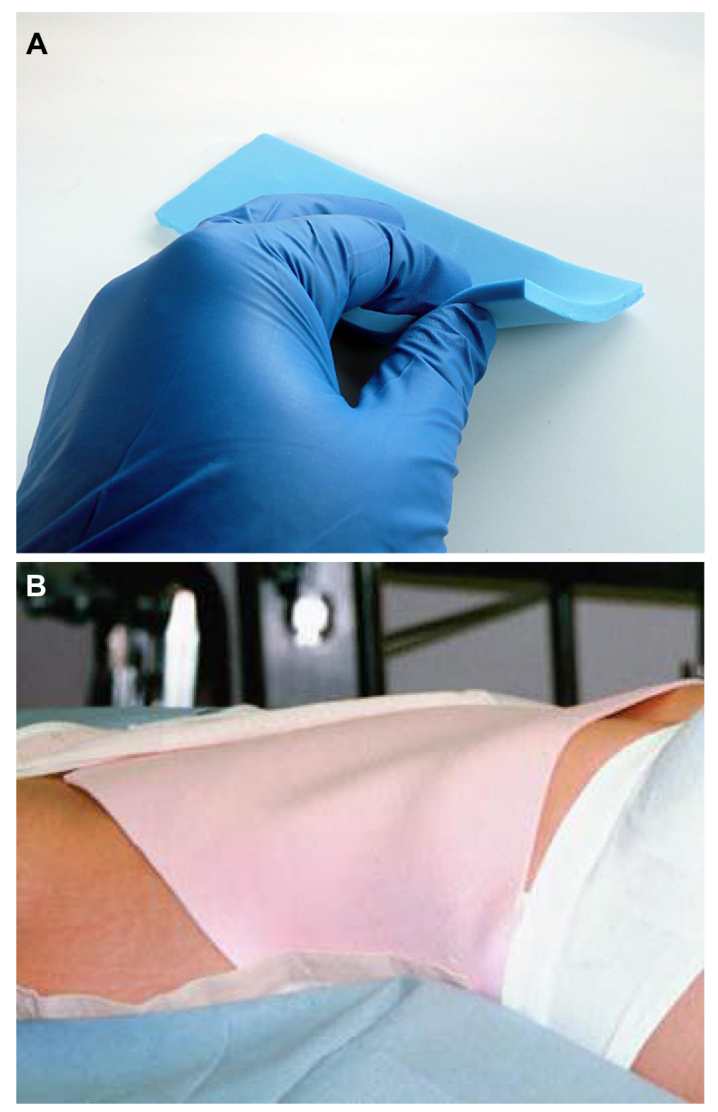

Figure 17 (A) Close-up of closed-cell compression foam (TenderFoam ${ }^{\mathrm{TM}}$; T\&N Industries, San Diego, CA, USA). (B) Foam in place prior to firm compression.
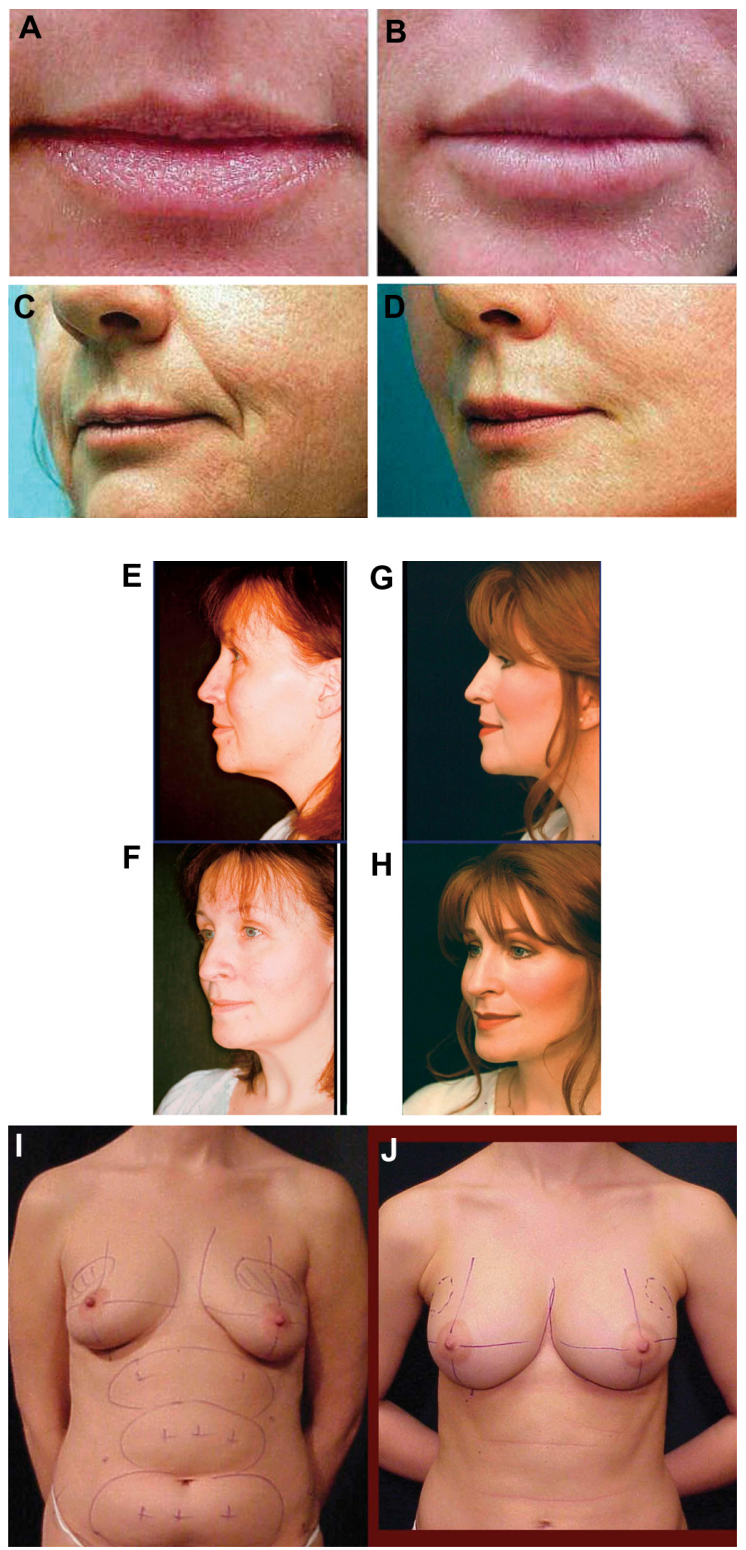

Figure 18 Clinical examples. Lips (A) pre- and (B) I-year post-augmentation (autologous fat grafting [AFG] plus high-density platelet-rich plasma [HD PRP] [upper lip 3 cc; lower lip 2 cc]). (C) Pre- and (D) postoperative (20 months) AFG plus HD PRP grafting to lips, cheeks and nasolabial folds (lips: upper $2.5 \mathrm{cc}$; lower $2 \mathrm{cc}$; cheeks: $5 \mathrm{cc}$ bilateral, malar and sub-malar placement; nasolabial folds: $3 \mathrm{cc}$ bilateral). Cheeks (E and $\mathbf{F})$ pre- and ( $\mathbf{G}$ and $\mathbf{H}) 2$ years post-AFG plus HD PRP (bilateral cheeks; total volume grafted, $5 \mathrm{cc}$ bilateral malar and sub-malar) (Photos with permission). (I) Pre- and (J) postoperative (4 years) large-volume augmentation of both breasts (closed-syringe technique, cell-friendly cannulas) using AFG plus HD PRP concentrate; right, 300 cc; left, 325 cc).

and the effects of the local microenvironment available with the recipient fat tissues.

The importance of signaling and growth factor secretion associated with certain paracrine effects, have changed the treatment paradigm of small volume structural grafting. While it is now clear that the loss of most of the mature adipocytes is inevitable, it is appreciated that they still play an important integral role, as they participate in 
stimulation of the microenvironment, within the associated stromal vascular fraction. The near-terminally differentiated pre-adipocytes are activated to initiate their final differentiation into adipocytes and contribution to lipid metabolism and volume storage. Further, it is now known that the cellular and extracellular matrix (ECM) associated within the stromal elements serves an equally important role, serving as a living bio-scaffold (attachment to adipocytes and SVF) that is critical in encouraging available multipotent cells to effectively activate and proliferate. Both the AFG and additive effects are further enhanced in the surgically damaged recipient tissues through complex "signaling" mechanisms of autocrine and paracrine pathways in vivo., ${ }^{4,36}$

\section{Conclusion}

This paper presents a simple and effective method of lipoaspiration with which to harvest adipocytes and their accompanying progenitor and stromal elements using a disposable, closed syringe and microcannula system. Effective for lipoaspiration of small and large volumes, the use of a closed-syringe system and its accessories offers a full range of options by which to fulfill all AFG requirements. The safety and efficacy of using closed-syringe systems has evolved to the use of a coated, disposable microcannula system specifically designed for use in structural autologous fat harvest and transfer, which currently serves as the most complete and effective gold standard for all closed-syringe systems.

\section{Acknowledgment}

All patients consented for use of photographs.

\section{Disclosure}

The authors report no conflicts of interest in this work. There are no financial conflicts of interest with Tulip Medical TM, Johnson \& Johnson (Telfa ${ }^{\mathrm{TM}}$ ), or T\&N Industries (TenderFoam $^{\mathrm{TM}}$ ). Dr Harrell is currently the Chief of Cellular Science and Education, Harvest-Terumo, Plymouth, MA, USA.

\section{References}

1. Alexander RW. Liposculpture in the superficial plane: closed syringe system for improvements in fat removal and free fat transfer. American Journal of Cosmetic Surgery. 1992;11:127-134.

2. Zuk PA, Zhu M, Mizuno H, et al. Multilineage cells from human adipose tissue: implications for cell-based therapies. Tissue Eng. 2001;7(2):211-238.

3. Zuk PA, Zhu M, Ashjian P, et al. Human adipose tissue is a source of multipotent stem cells. MolBiol Cell. 2002;13(12):4279-4295.
4. Alexander RW. Understanding adipose-derived stromal vascular fraction (AD-SVF) cell biology and use on the basis of cellular, chemical, structural and paracrine components: a concise review. Journal of Prolotherapy. 2012;4(1):e855-e869.

5. Alderman D, Alexander RW. Advances in regenerative medicine: high-density platelet-rich plasma and stem cell prolotherapy. Journal of Prolotherapy. 2011;10:49-90.

6. Suga $\mathrm{H}$, Eto $\mathrm{H}$, Aoi N, et al. Adipose tissue remodeling under ischemia: death of adipocytes and activation of stem/progenitor cells. PlastReconstr Surg. 2010;126(6):1911-1923.

7. Eto H, Suga H, Inoue K, et al. Adipose injury-associated factors mitigate hypoxia in ischemic tissues through activation of adipose-derived stem/progenitor/stromal cells and induction of angiogenesis. Am J Path. 2011;178(5):2322-2332.

8. Kato H. Short- and long-term cellular events in adipose tissue remodeling after non-vascularized grafting. Paper presented at the International Federation for Adipose Therapeutics and Science Miami 2011 9th Annual Symposium on Adipose Stem Cells and Clinical Applications of Adipose Tissue; 2011 Nov 4-6; Miami, Florida, USA.

9. Doi K. Cellular origin in adipose tissue remodeling after transplantation: host or donor? Paper presented at the International Federation for Adipose Therapeutics and Science Miami 2011 9th Annual Symposium on Adipose Stem Cells and Clinical Applications of Adipose Tissue; 2011 Nov 4-6; Miami, Florida, USA.

10. Sadati KS, Corrado AC, Alexander RW. Platelet-rich plasma (PRP) utilized to promote greater graft volume retention in autologous fat grafting. American Journal of Cosmetic Surgery. 2006;23(4): $627-631$.

11. Gimble JM, Katz AJ, Bunnell BA. Adipose-derived stem cells for regenerative medicine. Circ Res. 2007;100(9):1249-1260.

12. Coleman SR. Structural lipoaugmentation.In: Narins RS, editor. Safe Liposuction and Fat Transfer. New York, NY: Marcel Dekker; 2003:409-423.

13. Maumas M, Peyrafitte JA, D'Angelo R, et al. Native human adipose stromal cells: localization, morphology and phenotype. Intl J Obes (Lond). 2011;35(9):1141-1153.

14. Strem BM, Hicok KC, Zhu M, et al. Multipotential differentiation of adipose-derived stem cells. Keio J Med. 2005;54(3):132-141.

15. Parker AM, Katz AJ. Adipose-derived stem cells for the regeneration of damaged tissues. Expert OpinBiolTher.2006;6(6):567-578.

16. Guilak F, Cohen DM, Estes BT, Gimble JM, Liedtke W, Chen CS. Control of stem cell fate by physical interactions with the extracellular matrix. Cell Stem Cell.2009;5(1):17-26.

17. Guilak F, Estes BT, Diekman BO, Moutos FT, Gimble JM. 2010 Nicolas Andry Award: Multipotent adult stem cells from adipose tissue for musculoskeletal tissue engineering. ClinOrthopRelat Res.2010;468(9):2530-2540.

18. Albano J, Alexander RW. Autologous fat grafting as a mesenchymal stem cell source and living bioscaffold in a patellar tendon tear. $\mathrm{Am} \mathrm{J}$ Sports Med. 2011;21(4):359-361.

19. Alexander, Robert W.: Author Textbook, Use of PRP In Autologous Fat Grafting, in Autologous Fat Grafting, Textbook, Shiffman, M. ed., Springer, Berlin; 2010;14:87-112.

20. Alderman DD, Alexander RW, Harris GR. Stem cell prolotherapy in regenerative medicine: background, research and protocols. Journal of Prolotherapy, 2011;3(3):689-708.

21. Astori G, Vignati F, Bardelli S, et al. "In vitro" and multicolor phenotypic characterization of cell subpopulations identified in fresh human adipose tissue stromal vascular fraction and in the derived mesenchymal stem cells. J Translat Med. 2007;5(55):1-10.

22. Gimble JM, Bunnell BA, Builak F, Smith SR, Katz AJ. Isolation and growth of stem cells. In: Pallua N, Suscheck CV, editors. Tissue Engineering: From Lab to Clinic. Berlin: Springer-Verlag; 2011: 93-111.

23. Mizuno H. Adipose-derived stem and stromal cells for cell-based therapy: current status of preclinical studies and clinical trials. CurrOpinMolTher. 2010;12(4):442-449. 
24. Nixon AJ, Dahlgren LA, Haupt JL, Yeager AE, Ward DL. Effect of adipose-derived nucleated cell fractions on tendon repair in horses with collagenase-induced tendinitis. Am J Vet Res. 2008;69(7):928-937.

25. Yu G, Floyd ZE, Wu X, Halvorsen YD, Gimble JM. Isolation of human adipose-derived stem cells from lipoaspirates. Methods Mol Biol. 2011;702:17-27.

26. Black LL, Gaynor J, Gahring D, et al. Effect of adipose-derived mesenchymal stem and regenerative cells on lameness in dogs with chronic osteoarthritis of the coxofemoral joints: a randomized, double-blinded, multicenter, controlled trial. Vet Ther. 2007;8(4):272-284.

27. Uysal AC, Mizuno H. Tendon regeneration and repair with adipose derived stem cells. Curr Stem Cell Res Ther. 2010;5(2):161-167.

28. Prockop DJ. Marrow stromal cells as stem cells for nonhematopoietic tissues. Science. 1997;276(5309):71-74.

29. Alexander RW. Fat transfer with platelet-rich plasma for breast augmentation. In: Shiffman MA, editor. Breast Augmentation: Principles and Practice. Berlin: Springer; 2009:451-470.

30. Aust L, Devlin B, Foster S, et al. Yield of human adipose-derived adult stem cells from liposuction aspirates. Cytotherapy.2004;6(1):7-14.

31. Yoshimura K, Shigeura T, Matsumoto D, et al. Characterization of freshly isolated and cultured cells derived from the fatty and fluid portions of liposuction aspirates. J Cell Physiol. 2006;208(1):64-76.

32. Traktuev DO, Merfeld-Clauss S, Li J, et al. A population of multipotent CD34-positive adipose stromal cells share pericyte and mesenchymal surface markers, reside in a periendothelial location, and stabilize endothelial networks. Circ Res.2008;102(1):77-85.
33. Crisan M, Yap S, Casteilla L, et al. A perivascular origin for mesenchymal stem cells in multiple human organs. Cell Stem Cell. 2008;3(3):301-313.

34. Granneman JG, Li P, Lu Y, Tilak J. Seeing the trees in the forest: selective electroporation of adipocytes within adipose tissue. Am J PhysiolEndocrinolMetab. 2004;287(3):E574-582.

35. Spalding KL, Arner E, Westermark PO, et al. Dynamics of fat cell turnover in humans. Nature. 2008;453(7196):783-787.

36. Abuzeni PZ, Alexander RW. Enhancement of autologous fat transplantation with platelet rich plasma. American Journal of Cosmetic Surgery. 2001;18(2):59-70.

37. Alexander RW. Autologous fat grafts as mesenchymal stromal stem cell source for use in prolotherapy: a simple technique to acquire lipoaspirants. Journal of Prolotherapy.2011;3(3):680-688.

38. Yoshimura K, Sato K, Aoi N, Kurita M, Hirohi T, Harii K. Cell-assisted lipotransfer for cosmetic breast augmentation: supportive use of adipose-derived stem/stromal cells. AesthPlast Surg. 2008;32(1):48-55.

39. Kurita M, Matsumoto D, Shigeura T, et al. Influences of centrifugation on cells and tissues in liposuction aspirates: optimized centrifugation for lipotransfer and cell isolation. PlastReconstr Surg. 2008;121(3):1033-1041.

40. Alexander RW. Autologous fat grafting: a study of residual intracellular adipocyte lidocaine. In: Shiffman MA, editor. Autologous Fat Grafting: Art, Science, and Clinical Practice. Berlin and Heidelberg: SpringerVerlag; 2010:445-450.
Clinical, Cosmetic and Investigational Dermatology

\section{Publish your work in this journal}

Clinical, Cosmetic and Investigational Dermatology is an international, peer-reviewed, open access, online journal that focuses on the latest clinical and experimental research in all aspects of skin disease and cosmetic interventions. All areas of dermatology will be covered; contributions will be welcomed from all clinicians and

\section{Dovepress}

basic science researchers globally. This journal is indexed on CAS. The manuscript management system is completely online and includes a very quick and fair peer-review system, which is all easy to use. Visit http://www.dovepress.com/testimonials.php to read real quotes from published authors. 